\title{
Excited states of neutral donor bound excitons in GaN
}

Cite as: J. Appl. Phys. 123, 215702 (2018); https://doi.org/10.1063/1.5028370

Submitted: 09 March 2018. Accepted: 14 May 2018 . Published Online: 05 June 2018

G. Callsen, T. Kure, M. R. Wagner (D), R. Butté (D), and N. Grandjean (iD)
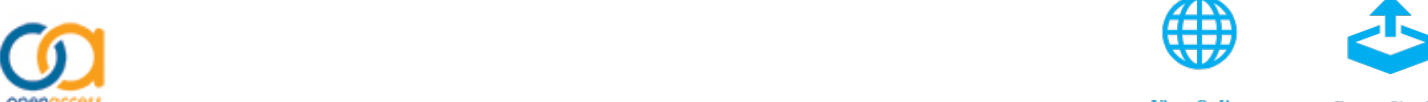

View Online

\section{ARTICLES YOU MAY BE INTERESTED IN}

GaN surface as the source of non-radiative defects in InGaN/GaN quantum wells Applied Physics Letters 113, 111106 (2018); https://doi.org/10.1063/1.5048010

Luminescence properties of defects in GaN

Journal of Applied Physics 97, 061301 (2005); https://doi.org/10.1063/1.1868059

Low injection losses in InGaN/GaN LEDs: The correlation of photoluminescence, electroluminescence, and photocurrent measurements

Journal of Applied Physics 123, 214502 (2018); https://doi.org/10.1063/1.5022026

Journal of Applied Physics
SPECIAL TOPIC:

\section{Antiferromagnetic Spintronics}




\title{
Excited states of neutral donor bound excitons in GaN
}

\author{
G. Callsen, ${ }^{1}$ T. Kure, ${ }^{2}$ M. R. Wagner, ${ }^{2}$ R. Butté,${ }^{1}$ and N. Grandjean ${ }^{1}$ \\ ${ }^{1}$ Institute of Physics, École Polytechnique Fédérale de Lausanne (EPFL), CH-1015 Lausanne, Switzerland \\ ${ }^{2}$ Institut für Festkörperphysik, Technische Universität Berlin, Hardenbergstraße 36, DE-10623 Berlin, \\ Germany
}

(Received 9 March 2018; accepted 14 May 2018; published online 5 June 2018)

\begin{abstract}
We investigate the excited states of a neutral donor bound exciton $\left(\mathrm{D}^{0} \mathrm{X}\right)$ in bulk $\mathrm{GaN}$ by means of high-resolution, polychromatic photoluminescence excitation (PLE) spectroscopy. The optically most prominent donor in our sample is silicon accompanied by only a minor contribution of oxygen - the key for an unambiguous assignment of excited states. Consequently, we can observe a multitude of $\mathrm{Si}^{0} \mathrm{X}$-related excitation channels with linewidths down to $200 \mu \mathrm{eV}$. Two groups of excitation channels are identified, belonging either to rotational-vibrational or electronic excited states of the hole in the $\mathrm{Si}^{0} \mathrm{X}$ complex. Such identification is achieved by modeling the excited states based on the equations of motion for a Kratzer potential, taking into account the particularly large anisotropy of effective hole masses in GaN. Furthermore, several ground- and excited states of the exciton-polaritons and the dominant bound exciton are observed in the photoluminescence (PL) and PLE spectra, facilitating an estimate of the associated complex binding energies. Our data clearly show that great care must be taken if only PL spectra of $\mathrm{D}^{0} \mathrm{X}$ centers in GaN are analyzed. Every PL feature we observe at higher emission energies with regard to the $\mathrm{Si}^{0} \mathrm{X}$ ground state corresponds to an excited state. Hence, any unambiguous peak identification renders PLE spectra highly valuable, as important spectral features are obscured in common PL spectra. Here, GaN represents a particular case among the wide-bandgap, wurtzite semiconductors, as comparably low localization energies for common $\mathrm{D}^{0} \mathrm{X}$ centers are usually paired with large emission linewidths and the prominent optical signature of exciton-polaritons, making the sole analysis of PL spectra a challenging task. (C) 2018 Author(s). All article content, except where otherwise noted, is licensed under a Creative Commons Attribution (CC BY) license (http://creativecommons.org/licenses/by/ 4.0/). https://doi.org/10.1063/1.5028370
\end{abstract}

\section{INTRODUCTION}

Close to the bandedge energy of a bulk semiconductor, one can frequently observe the intrinsic exciton emission lines in addition to a rich spectrum of bound exciton complexes-a unique window for monitoring different impurity species. Each individual type of impurity can bind an exciton, usually leading to a bright and distinct emission line in any direct bandgap semiconductor, lowered by the localization energy $\left(E_{l o c}\right)$ with regard to the intrinsic exciton emission due to a chemical shift. Since the early days of bound exciton spectroscopy, it was the aim to assign a particular impurity to each experimentally observed chemical shift, in order to utilize the spectrum of bound excitons as an impurity fingerprint. ${ }^{1}$ Even though bound exciton spectroscopy probes the presence of impurities well below the conventional detection limit of alternative techniques like, e.g., secondary ion mass spectroscopy (SIMS), ${ }^{2}$ any quantification of the impurity concentration often remains laborious due to varying free exciton capture cross sections among the impurities. $^{3}$

In order to obtain a solid relation between a bound exciton and an impurity one can, e.g., undertake photoluminescence (PL) measurements at low temperature on samples that contain radioactive isotopes of the dopant. ${ }^{4}$ For instance, ion implantation in combination with thermal annealing or thermal diffusion techniques has proven to yield samples suitable for such impurity studies. Exemplarily, Meyer et al. summarize such identification efforts in $\mathrm{ZnO},{ }^{1}$ which have led to the chemical identification of the most prominent neutral donor bound excitons $\left(\mathrm{D}^{0} \mathrm{X}\right)$, similar to comparable achievements in cubic $\mathrm{ZnSe},{ }^{5}$ and many other direct bandgap wurtzite semiconductors. ${ }^{2}$ For instance, in $\mathrm{ZnO}$, the most prominent $\mathrm{D}^{0} \mathrm{X}$ emission lines are found in an $E_{l o c}$ interval of around $35 \mathrm{meV}$, featuring emission linewidths well below $100 \mu \mathrm{eV} .{ }^{6}$ In contrast, in $\mathrm{GaN}$ the dominant $\mathrm{D}^{0} \mathrm{X}$ emission lines are only distributed over approximately $10 \mathrm{meV}$ with common emission linewidths around $1 \mathrm{meV}$ and only a very few reports achieving record linewidths of $100 \mu \mathrm{eV}{ }^{7}$ In addition, a straightforward analysis of bound excitons in $\mathrm{GaN}$ is often plagued by a number of excited $\mathrm{D}^{0} \mathrm{X}$ states that appear in luminescence on the high-energy tail of the $\mathrm{D}^{0} \mathrm{X}$ emission. ${ }^{8}$ Hence, it appears as a clear challenge to distinguish between additional bound exciton lines in their ground state and features of excited states due to peak overlap. In addition, most high quality GaN samples that were used for the pioneering analysis of the excited states of $\mathrm{D}^{0} \mathrm{X}$ centers $^{8,9}$ exhibited prominent bound excitonic emission related to oxygen and silicon of comparable intensity. Hence, as the $\mathrm{Si}^{0} \mathrm{X}$ and $\mathrm{O}^{0} \mathrm{X}$ ground state emission is only split by around $900 \mu \mathrm{eV},{ }^{8,10}$ it becomes extremely challenging to identify all the luminescence features related to the excited states, even for selectively excited PL. 
In contrast, our $\mathrm{GaN}$ sample only comprises a negligible luminescence contribution related to $\mathrm{O}^{0} \mathrm{X}$; hence, the entire near bandedge spectrum is dominated by $\mathrm{Si}^{0} \mathrm{X}$ facilitating an identification of all the associated excited states. In contrast to previous studies, ${ }^{9}$ we do not restrict our analysis to selectively excited PL spectra, but record polychromatic photoluminescence excitation (PLE) spectra-the technique of choice for analyzing excited states of excitonic complexes. ${ }^{11}$ On the one hand, above the energy of the A-exciton-polariton $\left(\mathrm{X}_{\mathrm{A}}\right)$ we observe a set of excitation channels related to the exciton-polaritons and their excited states $(n=2)$, which we also find to bind at the $\mathrm{Si}^{0}$ center. On the other hand, below the energy of $\mathrm{X}_{\mathrm{A}}$ we can identify up to six additional excited states, all related to the $\mathrm{Si}^{0} \mathrm{X}_{\mathrm{A}}$ center as supported by calculations. Furthermore, even excited states related to the B-valence band can be identified. Based on a consistent set of material parameters for $\mathrm{GaN}$, we can relate our experimental findings to vibrational-rotational excited states as well as electronically excited states of the hole in the $\mathrm{Si}^{0} \mathrm{X}_{\mathrm{A}}$ complex. Our results highlight that great care must be taken when identifying bound excitons and their excited states in GaN by PL, as the combination of low $E_{l o c}$ values and rather large linewidths renders PLE measurements the technique of choice.

\section{EXPERIMENTAL DETAILS}

The investigated GaN sample was grown on $c$-plane, random core, GaN substrates ${ }^{12}$ ( $n$-type) obtained from a hydride vapor phase epitaxy based overgrowth technique, with a dislocation density $\sim 10^{6} \mathrm{~cm}^{-2}$. First, a 360-nm-thick GaN buffer is grown at a temperature of $1000^{\circ} \mathrm{C}$ with tri-methylgallium and $\mathrm{H}_{2}$ as carrier gas. Subsequently, a 500-nm-thick $\mathrm{Al}_{0.06} \mathrm{Ga}_{0.94} \mathrm{~N}$ layer is deposited at $1000^{\circ} \mathrm{C}$ with tri-methylaluminum and $\mathrm{H}_{2}$ as carrier gas, before $100 \mathrm{~nm}$ of $\mathrm{GaN}$ is grown at a temperature of $950{ }^{\circ} \mathrm{C}$ based on a tri-ethyl-gallium precursor. The use of an $\mathrm{Al}_{0.06} \mathrm{Ga}_{0.94} \mathrm{~N}$ interlayer allows us to inhibit carrier migration and to study the top GaN layer optically without any significant luminescence contribution from the underlying GaN buffer and substrate. SIMS measurements confirmed that the oxygen and silicon concentration in our sample is below the detection limit $\left(\leq 2 \times 10^{16} \mathrm{~cm}^{-3}\right)$, while the carbon concentration amounts to $\sim 1 \times 10^{16} \mathrm{~cm}^{-3}$. Such a characteristic shall serve as a basis to reveal the negligible contribution of oxygen-related neutral donor bound excitonic features in our sample based on the PL and PLE analysis discussed in Secs. III A and V.

PL and PLE spectra were measured with the sample mounted in a cold-finger helium-flow cryostat at a temperature of $4 \mathrm{~K}$. We used a tunable dye laser as excitation source that employed 2-methyl-5-t-butyl-p-quaterphenyl as active medium dissolved in 1,4-dioxane for spectroscopic purposes. The dye laser was pumped by a $\mathrm{XeCl}$ excimer laser emitting at $308 \mathrm{~nm}$ with a repetition rate of $100 \mathrm{~Hz}$, enabling a pulse duration of $12 \mathrm{~ns}$ for the dye laser. In order to avoid any high excitation effects, the quasi-continuous-wave pumping power of the dye laser was limited to $0.2 \mu \mathrm{W}$. The linewidth of the excitation laser amounts to around $40 \mu \mathrm{eV}$ and the side-mode suppression ratio amounts to two orders of magnitude. The excitation light was guided towards the sample at an incident angle of $45^{\circ}$ with respect to the $c$-axis $(k$ $\rightarrow 45^{\circ} c$-axis), while being focused by an achromatic lens doublet for the ultraviolet (UV) spectral regime with a focal length of $20 \mathrm{~cm}$ (excitation spot size diameter: $\sim 100 \mu \mathrm{m}$ ). The sample's luminescence was collected in $k \| c$ geometry using a lens with a numerical aperture of 0.56 , before it was finally imaged onto a Spex 1404 double monochromator ( $85 \mathrm{~cm}$ focal length). This monochromator was equipped with two $1200 \mathrm{l} / \mathrm{mm}$ gratings $(500 \mathrm{~nm}$ blaze wavelength), which we employed in second order to achieve an optical resolution better than $50 \mu \mathrm{eV}$ in the wavelength regime of interest. Finally, the dispersed light was monitored with a UV-enhanced, charge-coupled-device (CCD) array from Princeton Instruments $(128 \times 1340$ pixels $)$. Hence, for each chosen excitation wavelength we were able to record a PL spectrum, enabling, so-called, polychromatic PLE scans. Due to the large detection area of the CCD detector, it was of utmost importance to employ a double monochromator as a sufficiently low stray light level had to be achieved in close energetic vicinity to the excitation laser line. Furthermore, the chosen excitation and detection geometry for the PLE scans was dictated by the aim to suppress most of the laser light from already entering into the detection system. All PL and PLE spectra were calibrated based on a mercury calibration lamp. Subsequently, wavelength was converted to energy without applying any correction for the refractive index of air in order to make the present results more comparable to the commonly available literature treating bound excitons in GaN. Application of such a vacuum correction would shift all in the following reported absolute peak energies by around $900 \mu \mathrm{eV}$ to lower energies.

\section{RESULTS}

Figure 1(a) shows a scheme of the most common groups of excited states of a $\mathrm{D}^{0} \mathrm{X}_{\mathrm{A}}$ center that commonly appear energetically below the $\mathrm{X}_{\mathrm{A}}$ transition. Taking the $\mathrm{D}^{0} \mathrm{X}_{\mathrm{A}}$ as the energetic reference, one can observe the related $\mathrm{D}^{0} \mathrm{X}_{\mathrm{B}}$ center at higher energies (orange). The measured splitting closely matches the A-B-valence band splitting in wurtzite semiconductors like $\mathrm{ZnO}^{13}$ and $\mathrm{CdS},{ }^{14,15}$ which is governed by the subtle balance between the crystal-field and the spinorbit interaction at such a shallowly bound impurity. In addition, electronic excited states (blue) can be observed in both PL and PLE spectra, which correspond to the excited (e.g., $p$ - and $d$-like) states of the hole in a $\mathrm{D}^{0} \mathrm{X}_{\mathrm{A}}$ center, as first described by Puls et al. for excited $\mathrm{D}^{0} \mathrm{X}_{\mathrm{A}}$ states in CdS. ${ }^{16}$ Similar to molecular vibrations, the four-particle complex of a $\mathrm{D}^{0} \mathrm{X}_{\mathrm{A}}$ center can also exhibit rotational and vibrational states (green), which are usually situated in close vicinity to the ground state. ${ }^{1,13}$ As first pointed out by Thomas and Hopfield for bound excitons in $\mathrm{CdS},{ }^{15}$ a clear experimental distinction between such electronic and rotational-vibrational excited states is not feasible as the electron and hole possess effective masses of the same order of magnitude-in clear contrast to the atomic/molecular case. Hence, in order to separate both types of excited states one has to rely on modeling as presented in Sec. IV. 


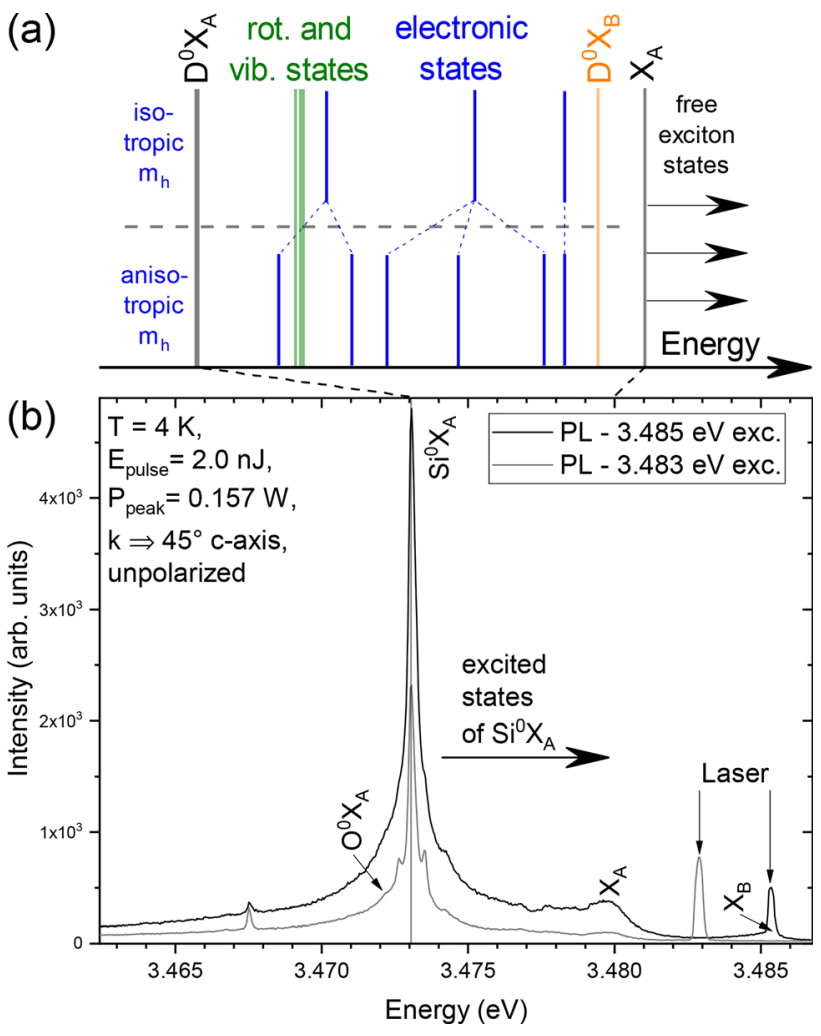

FIG. 1. (a) Sketch illustrating the two main groups of $\mathrm{D}^{0} \mathrm{X}_{\mathrm{A}}$-related excited states below the free A-exciton $\left(\mathrm{X}_{\mathrm{A}}\right)$ : rotational-vibrational states (green) as well as electronic excited states (blue and orange). Here, $\mathrm{D}^{0} \mathrm{X}_{\mathrm{B}}$ denotes a neutral donor bound exciton comprising a hole from the B-valence band. The large anisotropy in the GaN hole mass $m_{h}$ lifts the degeneracy of excited states, leading to optically resolvable splittings for the case of electronic excited states. The PL spectra in (b) were either excited close to resonance with $X_{B}$ (black) or in between the $X_{A}$ and $X_{B}$ transitions (grey). Such selective excitation allows resolving parts of the excitonic fine structure as introduced in (a).

Depending on the anisotropy of the hole mass $m_{h}$, the degeneracy of the electronic states with respect to the magnetic quantum number $(m)$ is lifted, leading to, e.g., a twoor threefold splitting depending on the angular momentum quantum number $l .{ }^{16}$ Normally, states with $l \geq 2$ lie energetically above the $\mathrm{X}_{\mathrm{A}}$ transition and are therefore unstable. Usually, the influence of the hole mass anisotropy on the rotational-vibrational states cannot be resolved by emissionbased techniques like PL and PLE and would for instance rather require infrared absorption measurements. ${ }^{17,18}$

In order to summarize the experimental challenge, it is important to note that all these different groups of excited states are spectrally situated in between the emission energy of $\mathrm{X}_{\mathrm{A}}(\approx 3.480 \mathrm{eV})$ and $\mathrm{Si}^{0} \mathrm{X}_{\mathrm{A}}(\approx 3.473 \mathrm{eV})$ at a temperature of $4 \mathrm{~K}$ as highlighted in Fig. 1. Hence, in $\mathrm{GaN}$ a large number of excited states are just distributed over an energy window of around $7 \mathrm{meV}$, rendering PLE measurements wellsuited, as they provide the strongest isolation from extraneous excitation processes leading to emission line broadening and peak overlap. ${ }^{11}$

\section{A. Basics of excited states related to $D^{0} X_{A}$ centers}

Because of the numerous excited states of a $\mathrm{D}^{0} \mathrm{X}_{\mathrm{A}}$ center, the spectrum recorded close to resonant excitation of $X_{B}$ displayed in Fig. 1(b) (3.485 eV excitation-black) shows a strongly broadened but still structured emission tail towards higher energies, rendering it extremely difficult to pinpoint certain transitions. However, as soon as the excitation laser is tuned in between the $\mathrm{X}_{\mathrm{A}}$ and $\mathrm{X}_{\mathrm{B}}$ transition $(3.483 \mathrm{eV}$ excitation-grey), i.e., towards lower absorption, one starts to observe finer details, which is accompanied by an emission linewidth reduction from around 400 to $200 \mu \mathrm{eV}$. Clearly, the lower absorption in between $\mathrm{X}_{\mathrm{A}}$ and $\mathrm{X}_{\mathrm{B}}$ increases the degree of details visible in the spectra, as the excitation of $\mathrm{Si}^{0} \mathrm{X}_{\mathrm{A}}$ is less dominated by exciton transfer towards the binding center in favor of a direct excitation of the bound exciton via the quasi continuum of electronic excited states $(l \geq 2){ }^{11}$ Interestingly, the optical signature related to the $\mathrm{O}^{0} \mathrm{X}_{\mathrm{A}}$ center-about $900 \mu \mathrm{eV}$ lower in energy compared to $\mathrm{Si}^{0} \mathrm{X}_{\mathrm{A}}{ }^{8,10,19}$ - is very low in intensity, representing a significant simplification for the analysis of our PL and PLE spectra, as we will not have to deal with two sequences of overlaying excited states. In addition, the PL spectra in Fig. 1(b) show an emission line at $3.4675 \mathrm{eV}$ of unknown origin. Several interpretations for this emission line exist in the literature ranging from a deep, over an ionized donor-bound exciton, to a neutral, shallow acceptor bound exciton. ${ }^{20-23}$ Even though this line is reported in the literature to appear at slightly different energies, its identification-but not its interpretation — can be achieved based on an apparent zero-field splitting on the order of $800 \mu \mathrm{eV}$ (not shown). ${ }^{22}$ However, a detailed PLE study of this comparably faint emission line remains a task for future work; at first, the basic excited states of a $\mathrm{D}^{0} \mathrm{X}_{\mathrm{A}}$ in GaN must be understood.

Figure 2 shows an overview PLE spectrum of the $\mathrm{Si}^{0} \mathrm{X}_{\mathrm{A}}$ complex (red) in addition to a PL spectrum (black) recorded with an excitation energy above the $\mathrm{C}$-exciton-polariton $\left(\mathrm{X}_{\mathrm{C}}\right)$ in $\mathrm{GaN}$ in order to approach the band continuum-an excitation condition similar to what is obtained by most groups with above bandgap excitation. Both spectra were extracted from the polychromatic PLE scans that include a PLE spectrum for each PL energy and vice versa. Here, Fig. 2 shows a PLE spectrum for a single pixel of the PL spectrum at the energetic position of the $\mathrm{Si}^{0} \mathrm{X}_{\mathrm{A}}$ transition corresponding to a bandpass of around $40 \mu \mathrm{eV}$, in good agreement with the spectral resolution of our spectrometer. In contrast, the step-width of the excitation laser was set to $105 \mu \mathrm{eV}$ in order to allow recording the present overview PLE spectrum in a reasonable timeframe, while the full-width at half maximum of the excitation laser was constant at around $40 \mu \mathrm{eV}$. Hence, depending on the settings, the PL and PLE resolution in all spectra are not always identical. In Fig. 2, we can now directly observe excitation channels of the $\mathrm{Si}^{0} \mathrm{X}_{\mathrm{A}}$ complex above (I) and below (II) the $\mathrm{X}_{\mathrm{A}}$ transition as introduced in Fig. 1. I: Towards higher energies, one first observes an excitation channel related to exciton-polaritons that comprise a hole from the $\mathrm{B}$-valence band $\left(\mathrm{X}_{\mathrm{B}}\right)$. Hence, we can estimate the $\mathrm{X}_{\mathrm{A}}-\mathrm{X}_{\mathrm{B}}$-splitting [5.5(1) meV-numbers in brackets indicate the error]. Even closer to the bandedge, the first excited states of these two transitions can be observed, exhibiting an almost identical splitting of 17.2(1) $\mathrm{meV}$ and $17.3(1) \mathrm{meV}$ (summarized in Table I) for $\mathrm{X}_{A}^{n=2}$, respectively, $\mathrm{X}_{B}^{n=2}$, due to the small corresponding variation of the effective exciton Rydberg. ${ }^{24}$ In addition, 21.6(2) $\mathrm{meV}$ above the $\mathrm{Si}^{0} \mathrm{X}_{\mathrm{A}}$ ground 


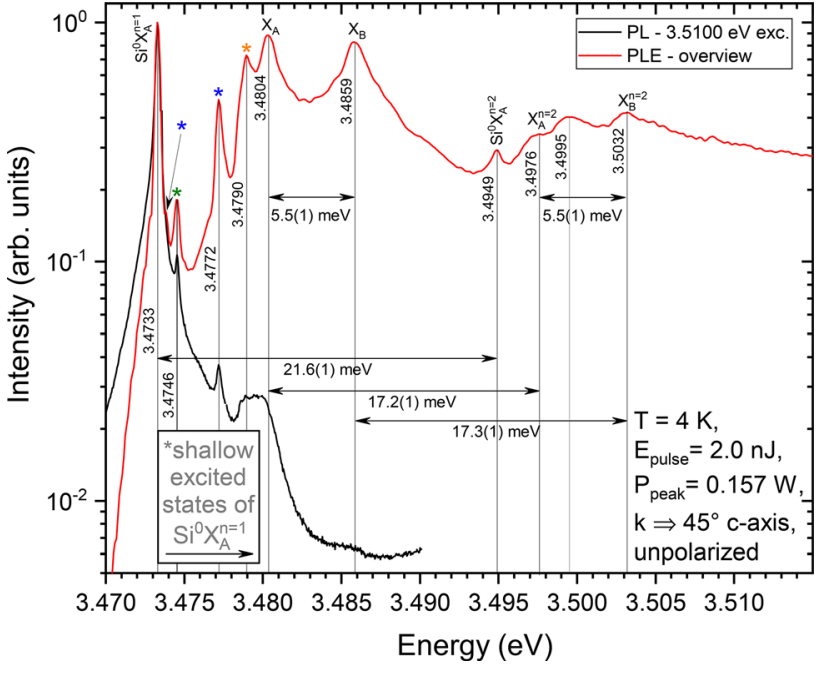

FIG. 2. Overview PLE spectrum (red) of a GaN sample compared with the corresponding PL spectrum (black). Energetically above the $\mathrm{X}_{\mathrm{A}}$ transition, one can observe a multitude of transitions related to excited states of free excitons, comprising a hole from the B-valence band $\left(\mathrm{X}_{\mathrm{B}}\right)$ and/or an electron in its first excited state $\left(\mathrm{X}_{A}^{n=2}\right.$ and $\left.\mathrm{X}_{B}^{n=2}\right)$. Transitions marked with an asterisk correspond to rotational-vibrational states (green), electronic excited states of the hole in the $\mathrm{D}^{0} \mathrm{X}_{\mathrm{A}}$ complex (blue), or excited states comprising a hole from the B-valence band (orange). Relevant energy splittings are highlighted. Numbers in brackets indicate the errors.

state the corresponding $\mathrm{Si}^{0} \mathrm{X}_{A}^{n=2}$ state appears in the PLE spectrum of Fig. 2, allowing us to estimate a silicon donor electron binding of 28.8(2) meV based on a simple effective mass approach (EMA). The transition noticeable in between $\mathrm{X}_{A}^{n=2}$ and $\mathrm{X}_{B}^{n=2}$ in Fig. 2 can tentatively be assigned to $\mathrm{X}_{\mathrm{C}}$, which mainly comprises a mixture of $\Gamma_{1}$ and $\Gamma_{5}$ states due to the selected PLE configuration. Here, the selection rules are relaxed due to the large numerical aperture of 0.56 of the collection lens and the incidence angle of the excitation laser of 45 degrees, facilitating the quasi-resonant excitation.

II: Towards lower energies with respect to $\mathrm{X}_{\mathrm{A}}$, one observes an entire set of excited states of the $\mathrm{Si}^{0} \mathrm{X}_{\mathrm{A}}$ complex marked with asterisks, which are either of an electronic (blue), or rotational-vibrational type (green). However, more detailed PLE measurements are required in order to obtain an assignment of all these excited states, which are also partially visible in the PL spectrum, cf. Fig. 2.

In addition to the determination of the silicon donor binding energy based on the splitting between $\mathrm{Si}^{0} \mathrm{X}_{A}^{n=2}$ and the $\mathrm{Si}^{0} \mathrm{X}_{A}^{n=1}$ ground state, we can estimate it based on the two-electron satellite (TES) transition, which was simultaneously recorded with the spectra from Fig. 2 (not shown). In these TES spectra, we do not observe any contribution of $\mathrm{O}^{0} \mathrm{X}_{\mathrm{A}}$-related $2 s$ - and $2 p$-states, rendering the spectroscopic analysis of the $\mathrm{Si}^{0} \mathrm{X}_{\mathrm{A}}$-related TES states a straightforward task. Here, we follow an approach described by Meyer $e t$ al. for the case of $\mathrm{ZnO},{ }^{1}$ which takes into account the different chemical shift for ground- and excited-states, but also polaron, as well as anisotropy corrections. ${ }^{24-26}$ Hence, based on a $1 s$ to $2 p_{z}$ splitting of $19.95(10) \mathrm{meV}$ we can determine a silicon donor binding energy of $27.3(5) \mathrm{meV}$. This value is significantly smaller than the silicon donor binding energy we obtained based on the $\mathrm{Si}^{0} \mathrm{X}_{A}^{n=1,2}$ states visible in Fig. 2 [28.8(2) meV]. This discrepancy of $1.5 \mathrm{meV}$ can be well understood based on the different and comparably large chemical shifts that occur for the $1 s$ and $2 s$ states in contrast to the less affected $2 p_{z}$ and $2 p_{x, y}$ states. A TES analysis based on such $1 s$ and $2 s$ states would yield a donor binding energy of 29.0(5) $\mathrm{meV}$, which is in reasonable agreement with the former more simplistic analysis of $\mathrm{Si}^{0} \mathrm{X}_{A}^{n=1,2}$ based on the PLE spectrum. However, these values always overestimate the donor binding energy as they are based on an oversimplified EMA, ${ }^{1}$ rendering the value of $27.3(5) \mathrm{meV}$ for the donor binding energy of silicon based on the $1 s$ to $2 p_{z}$ splitting the most reliable value we can obtain. Note that this silicon donor binding energy will form the basis for the calculations presented in Sec. IV. However, even deviations of a few $\mathrm{meV}$ will neither change the meaningfulness of the calculations nor the following interpretation.

\section{B. Detailed analysis of excited states with energies below the $\mathrm{X}_{\mathrm{A}}$ transition}

Figure 3 shows a high-resolution PLE spectrum of the $\mathrm{Si}^{0} \mathrm{X}_{A}^{n=1}$ center (red) along with a PL spectrum (black) for an excitation energy in between $\mathrm{X}_{A}^{n=1}$ and $\mathrm{X}_{B}^{n=1}$. In addition, a PLE spectrum for the low-energy side shoulder of the main $\mathrm{Si}^{0} \mathrm{X}_{A}^{n=1}$ emission is shown (purple). Starting at higher excitation energies, we first observe the excitation channels related to $X_{A}^{n=1}$ and $X_{B}^{n=1}$. However, in contrast to Fig. 2, a clear splitting is resolved. We attribute these splittings (see Table I) to the longitudinal-transverse splittings $(\Delta L T)$ of the $\Gamma_{5}$-exciton-polaritons related to the $\mathrm{A}-\left(\mathrm{X}_{\mathrm{A}}^{n=1(\mathrm{~L}, \mathrm{~T})}\right)$ and B-valence band $\left(\mathrm{X}_{\mathrm{B}}^{n=1(\mathrm{~L}, \mathrm{~T})}\right)$ in $\mathrm{GaN} .{ }^{27}$ Due to our particular experimental geometry $\left(k \rightarrow 45^{\circ} c\right.$-axis, no polarizers used) the selection rules are relaxed, hence leading to the partial observation of mixed exciton-polariton modes and thus the given approximation of $\Delta L T{ }^{28}$ The polarization- and angulardependent analysis of the optical signatures of the $\Gamma_{5^{-}}$and $\Gamma_{1}$-exciton-polaritons in this $\mathrm{GaN}$ sample should be left for future work. All relevant splittings are summarized in Table I.

In addition, at lower energy we observe a triplet peak structure centered $\sim 5.55(5) \mathrm{meV}$ in Fig. 3 (orange markers). We attribute this feature to the $\mathrm{Si}^{0} \mathrm{X}_{B}^{n=1}$ excitation channel of the ground state, not only based on the apparent similarity with the PLE and PL spectra in close energetic vicinity to $\mathrm{Si}^{0} \mathrm{X}_{A}^{n=1}$ (peak triplet), but also on a ground state to excited state splitting of 5.55(5) $\mathrm{meV}$. This value nicely matches the energy separation between $\mathrm{X}_{A}^{n=1}$ and $\mathrm{X}_{B}^{n=1}$, i.e., the A-B

TABLE I. Summary of the most relevant peak splittings extracted from Figs. 2 and 3 depending on the precision that could be reached during the peak position readout. $\Delta L T$ stands for the longitudinal-transverse splitting of the exciton-polaritons. Numbers in brackets indicate the errors. All numbers are given in meV.

\begin{tabular}{lccccccc}
\hline \hline $\mathrm{X}_{\mathrm{B}}^{n=1(\mathrm{~L})}-\mathrm{X}_{\mathrm{A}}^{n=1(\mathrm{~L})}$ & $\mathrm{X}_{\mathrm{B}}^{n=1(\mathrm{~T})}-\mathrm{X}_{\mathrm{A}}^{n=1(\mathrm{~T})}$ & $\Delta \mathrm{LT} \mathrm{X} \mathrm{A}^{n=1}$ & $\Delta \mathrm{LT} \mathrm{X}_{\mathrm{B}}^{n=1}$ & $\mathrm{X}_{\mathrm{A}}^{\mathrm{L}, n=2}-\mathrm{X}_{\mathrm{A}}^{\mathrm{L}, n=1}$ & $\mathrm{X}_{\mathrm{B}}^{\mathrm{L}, n=2}-\mathrm{X}_{\mathrm{B}}^{\mathrm{L}, n=1}$ & $\mathrm{Si}^{0} \mathrm{X}_{\mathrm{A}}^{n=2}-\mathrm{Si}^{0} \mathrm{X}_{\mathrm{A}}^{n=1}$ & $\mathrm{Si}^{0} \mathrm{X}_{\mathrm{B}}^{n=1}-\mathrm{Si}^{0} \mathrm{X}_{\mathrm{A}}^{n=1}$ \\
\hline $5.4(1)$ & $4.8(1)$ & $0.7(1)$ & $1.3(1)$ & $17.2(1)$ & $17.3(1)$ & $21.6(2)$ & $5.55(5)$ \\
\hline \hline
\end{tabular}




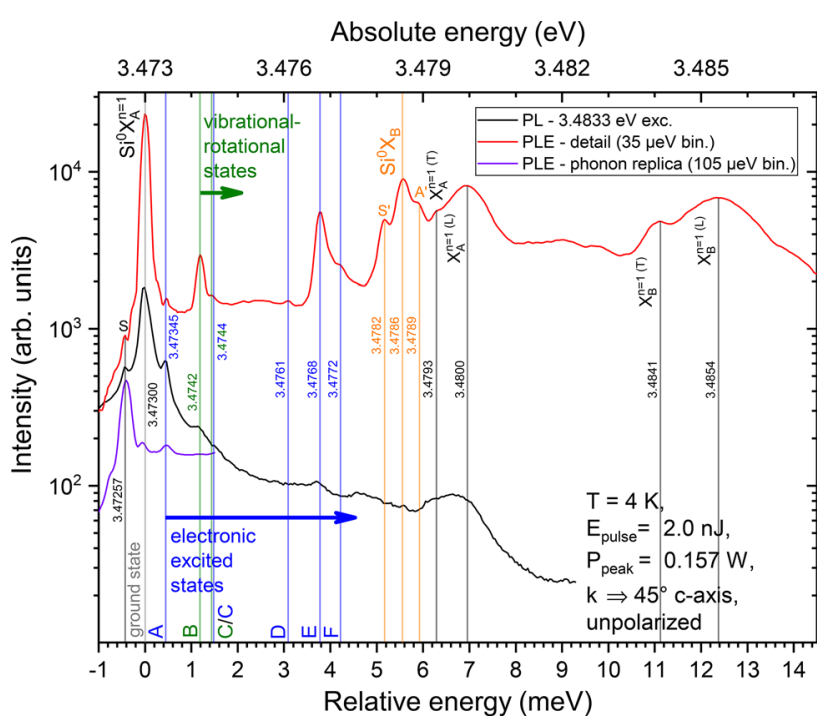

FIG. 3. High-resolution PLE spectrum (red line) showing the longitudinaltransverse-splitting of the A- and B-exciton-polaritons (black markers), the $B$ valence band related excited states of the neutral silicon donor bound exciton (orange markers), its electronic excited states (blue markers), and the associated vibrational-rotational states (green markers). The PL spectrum (black line) shows luminescence features for most of the excited states. In addition, a PLE spectrum recorded in the low-energy shoulder $\mathrm{S}$ of the $\mathrm{Si}^{0} \mathrm{X}_{A}^{n=1}$ transition (purple line) is shown in order to highlight the connection in between the ground state transition and its first electronic excited state A. The energies of the optical transitions A-F are calculated in Sec. IV and summarized in the term scheme of Fig. 4.

valance band splitting of GaN, cf. Table I. Former observations of this excitation channel in, e.g., $\mathrm{ZnO}$ and $\mathrm{CdS},{ }^{14,15}$ always yielded an energy separation that closely matched the $\mathrm{A}-\mathrm{B}$ valence band splitting, with the $\mathrm{I} 4$ line in $\mathrm{ZnO}$ as the only exception exhibiting a minor deviation of $0.4 \mathrm{meV} .^{13}$ The PL features related to the $\mathrm{Si}^{0} \mathrm{X}_{B}^{n=1}$ excitation channel triplet are faint and partially overlapping with the luminescence related to the A-exciton-polaritons. Furthermore, due to the small deviation of the hole masses related to the A- and B-valence band, it is natural to assume that $\mathrm{Si}^{0} \mathrm{X}_{B}^{n=1}$ features excited states similar to the ground state; hence, the apparent similarities between the related PL and PLE signatures. In Sec. V, the excitation and/or luminescence peak triplet related to $\mathrm{Si}^{0} \mathrm{X}_{A, B}^{n=1}$ will be discussed in more detail.

Lower in energy one also observes a sequence of at least six excitation channels (Fig. 3), which are labelled from A to $\mathrm{F}$ (green and blue) in the following. The most prominent PLE features are all well reproduced in the PL spectrum of Fig. 3, where they intensify with reducing energy spacing to $\mathrm{Si}^{0} \mathrm{X}_{A}^{n=1}$. The excitation channels $\mathrm{A}-\mathrm{F}$ should either be related to electronic and/or rotational-vibrational states as schematically introduced in Fig. 1. In order to obtain an assignment of those excitation channels, we calculate these excited states using a consistent set of material parameters for $\mathrm{GaN}^{24}$ as described in Sec. IV.

\section{CALCULATION OF THE EXCITED STATES}

In analogy to molecular vibrations, we first summarize an estimate of the rotational and vibrational energies of the $\mathrm{Si}^{0} \mathrm{X}_{A}^{n=1}$ exciton. Commonly, within a "pseudo-donor model" one assumes that the hole of the exciton is tightly bound to the acceptor species, ${ }^{29,30}$ while the electron is in a large orbit. In contrast, for a neutral donor bound exciton it is the hole, which exhibits a large orbit governed by the donor potential, giving rise to the excited states observed in experiments. Based on the simplification implied by replacing a Morse potential by a Kratzer potential ${ }^{31}$ in order to approximate the donor potential

$$
V(r)=-2 D\left(\frac{a}{r}-\frac{a^{2}}{2 r^{2}}\right),
$$

one can derive the following expression ${ }^{32}$ for the energy of the vibrational and rotational states with the corresponding vibrational and rotational quantum numbers $\nu$ and $J$ :

$$
E(\nu, J)=-\frac{\left(2 m_{h} a^{2} / \hbar^{2}\right) D^{2}}{\left[\left(\nu+\frac{1}{2}\right)+\sqrt{\left(J+\frac{1}{2}\right)^{2}+\left(\frac{2 m_{h} a^{2}}{\hbar^{2}}\right) D}\right]^{2}}
$$

Here, $m_{h}$ is the reduced hole mass averaged over the three directions in $k$-space, $a$ is the distance between the hole and the donor impurity, and $D$ is the donor binding energy as derived in Sec. III A. A first approximation of the interval for the parameter $a$ can be obtained from a simple EMA based on a typical magnesium acceptor binding energy in $\mathrm{GaN}$ ranging in between 150 and $200 \mathrm{meV}$, consequently leading to an $a$ value in between 0.5 and $0.4 \mathrm{~nm} .^{33,34}$

The energy of each individual vibrational and rotational state above the ground state $\mathrm{Si}^{0} \mathrm{X}_{A}^{n=1}$ is calculated as the difference between $E(\nu, J)$ and $E(0,0)$. Consequently, we obtain three groups of states: I. Two, energetically almost degenerate states $\{\nu, J\}=\{0,1\}=\{1,0\}$, II. Three degenerate states $\{\nu, J\}=\{0,2\}=\{1,1\}=\{2,0\}$, and III. A quasi-continuum of states with higher quantum numbers $\nu$ and $J$. In the following, we will always employ such grouping of states if their splitting cannot be resolved by the employed experimental means $(\sim 40 \mu \mathrm{eV})$. For the acceptor binding energy interval under consideration, the energy for the states in group I $\left(\mathrm{E}_{\mathrm{I}}\right)$ scales in between 1.44 and $0.87 \mathrm{meV}$, while we obtain $1.73 \mathrm{meV}$ to $1.05 \mathrm{meV}$ for group II $\left(\mathrm{E}_{\mathrm{II}}\right)$. These results already reach a good agreement with the experimental results shown in Fig. 3 regarding the excitation channels B and $\mathrm{C}$ (green) and the corresponding luminescence trace. Clearly, no other excitation channels occur within the calculated energy range for the rotationalvibrational states, rendering a misinterpretation unlikely. Interestingly, the best agreement between the experiment and theory is found for $a \approx 0.45 \mathrm{~nm}$, a value that would correspond to a relatively shallow magnesium acceptor binding energies $\left(E_{\text {bind }}\right)$ of, e.g., $164 \pm 5 \mathrm{meV}$ as experimentally determined by Callsen et al. ${ }^{33}$ for a GaN sample with a magnesium concentration of $8 \times 10^{17} \mathrm{~cm}^{-3}$. Hence, for this particular distance in between the hole and the donor impurity, we calculate an energy of $1.24 \mathrm{meV}$ for group $\mathrm{I}\left(\Delta \mathrm{E}_{\mathrm{I}}\right.$ $\approx 20 \mu \mathrm{eV})$, while group II is split-off by $250 \mu \mathrm{eV}\left(\Delta \mathrm{E}_{\mathrm{II}}\right.$ $\approx 10 \mu \mathrm{eV})$. Not only the absolute energy of these two groups 
of states achieves a good match with the experimental results as summarized in Fig. 4, also the energy splitting between the two groups is in good agreement. Finally, additional PLE excitation channels are observed in Figs. 3(a) and 3(d)-3(f) that cannot be explained by any rotational-vibrational excited states of $\mathrm{Si}^{0} \mathrm{X}_{A}^{n=1}$ and are discussed in the following.

Puls et al. have developed a theoretical model for neutral donor bound excitons in wurtzite $\mathrm{CdS}$ that allows the description of such additional excitation channels. ${ }^{16}$ These excited states of the $\mathrm{Si}^{0} \mathrm{X}_{A}^{n=1}$ exciton are related to $p$ - and $d$ like hole states that are fully characterized by the radial quantum number $n_{r}, l$, and $m$. By solving the Schrödinger equation of the neutral donor bound exciton complex considering the Kratzer potential from Eq. (1), one obtains an expression for the total energy $E(b)$ of each excited hole state $^{16}$

$$
\begin{aligned}
E(b)= & E_{g}+2 D\left[b^{2}-\frac{11}{8} b\right. \\
& \left.-\frac{s^{2} t^{2}}{2} \frac{m_{h}}{m_{e}}\left(n_{r}+\frac{1}{2}+\sqrt{\left(l+\frac{1}{2}\right)^{2}+\frac{s t^{2}}{b} \frac{m_{h}}{m_{e}}}\right)^{-2}\right] .
\end{aligned}
$$

Here, $b=\frac{a_{D}}{a_{e}}$ denotes the variational parameter used to minimize Eq. (3), comprising the fixed Bohr radius of the donor electron $a_{D}$ and an adjustable Bohr radius $a_{e}$ for the $1 s$ electron in the exciton bound to the $\mathrm{Si}^{0}$ impurity. Furthermore, $E_{g}$ describes the bandgap and $m_{e}$ is the reduced electron mass, while $s=1.0136$ and $t=1.337$ are related to the Kratzer potential and should be generally valid for excitonimpurity complexes. ${ }^{13}$ All other symbols have the same meaning as in Eq. (2). The electronic excited states calculated based on Eq. (3) still exhibit a degeneracy in $m$, which is lifted as soon as the anisotropy of the effective hole mass in $\mathrm{GaN}$ is considered. Depending on the particular excited state $\left|n_{r}, l, m\right\rangle, \quad$ a corresponding averaging of the perpendicular and parallel $m_{h}$ values must be considered, ${ }^{16}$ which is additionally weighted by the ratio of the static dielectric constants for each such directions. ${ }^{24}$

Interestingly, in the literature Eq. (3) is employed in two different ways: (1) the total energy $E(b)$ is either fully minimized $^{31}$ (technically correct) or (2) $E(b)$ is just minimized until the experimentally observed ground state energy is reached, ${ }^{13,16}$ while sometimes even $D$ is used as an additional free parameter. ${ }^{35}$ Based on our analysis from Sec. III A, we can utilize the experimentally determined, fixed D value of 27.3(5) meV. As a result, we obtain two differently spaced and ordered hierarchies of electronic states (black and red) as illustrated in Fig. 4 along with the rotational-vibrational excited states (green) and the experimental results (blue and orange). The calculation of electronic excited states based on approach (1) as shown in the right-hand side of Fig. 1 (red and black) predicts two experimentally observable PLE excitation channels if one considers the anisotropy of $m_{h}$ : the $|0,1,0\rangle$ state and an experimentally not resolvable overlap of $|0,1, \pm 1\rangle$ and $|0,2,0\rangle$ states. However, the number of observed shallow excitation channels $(\mathrm{A}-\mathrm{F})$ in PLE is significantly larger (Fig. 4).

Interestingly, based on approach (2) one can compute a much larger number of excited states of $\mathrm{Si}^{0} \mathrm{X}_{A}^{n=1}$ in the energy range of the experimental excitation channels. We believe that a similar observation for $\mathrm{ZnO}^{13}$ and $\mathrm{CdS}^{16}$ originates from the frequent application of method (2) in the literature directly illustrating a short-coming of the modeling related to the particular shape of the donor potential. Nevertheless, based on the left-hand side of Fig. 4 (black and red) we can obtain a tentative interpretation for the excitation channels A-F. The only transition energetically close to the excitation channel $\mathrm{A}$ is the $|0,1,0\rangle$ one, representing the rare case of an electronic state that is shifted below the rotational-vibrational states due to the large anisotropy of $m_{h}$ in GaN. Both excitation channels B and $\mathrm{C}$ seem to match with the two main groups of rotational-vibrational states. However, a degeneracy with the electronic states $|0,2,0\rangle$ and $|0,1, \pm 1\rangle$ cannot be excluded. The PLE features D-F

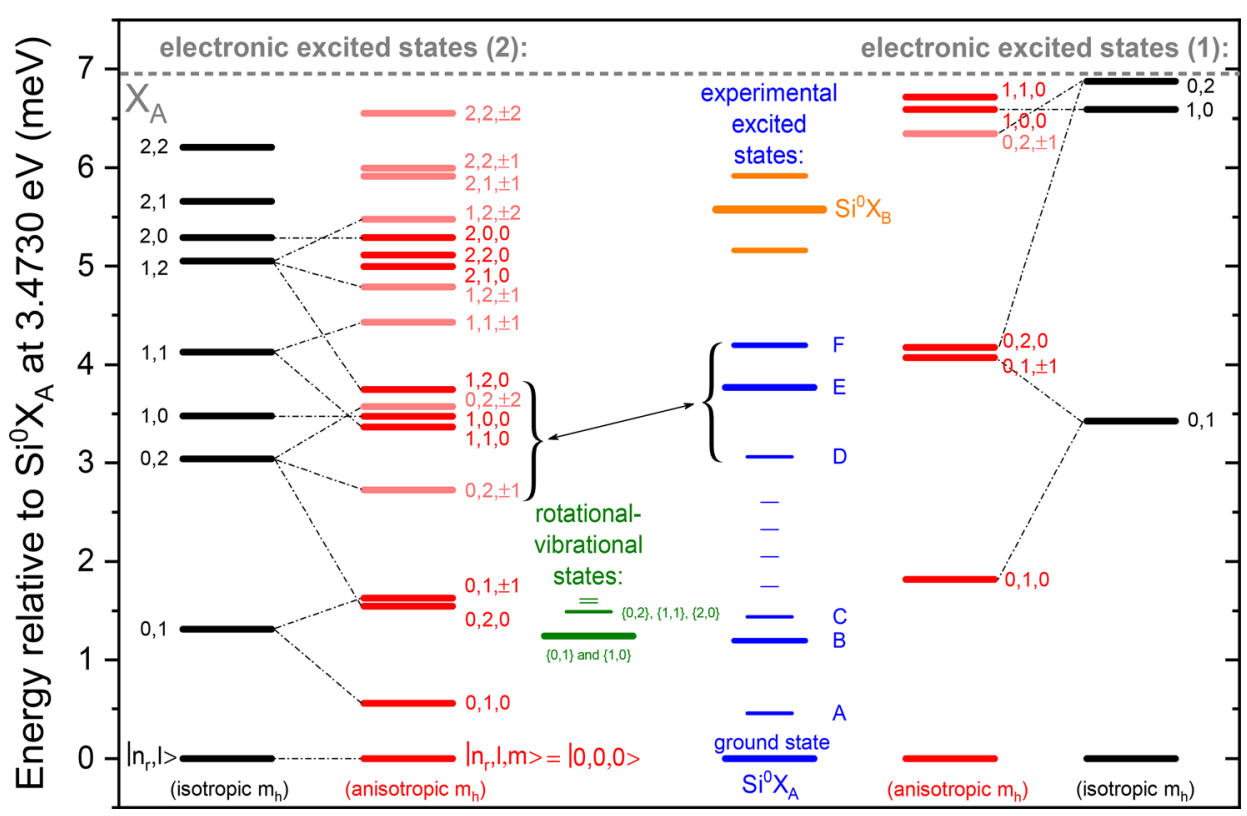

FIG. 4. Summary of all the excited states related to an exciton bound to the neutral silicon donor in GaN. The degeneracy in the magnetic quantum number $m$ is lifted for the electronic states (black) due to an anisotropy of the reduced hole mass $m_{h}$ leading to a large set of states (red). In addition, the calculated rotational-vibrational states are illustrated (green) along with the experimental results extracted from Fig. 3. The modeling is either based on (1) a full minimization of the total energy $E(b)$ or (2) on a minimization of $E(b)$ until the experimental ground state energy of $\mathrm{Si}^{0} \mathrm{X}_{\mathrm{A}}$ is reached. 
seem to correspond to the set of five states ranging from $|0,2, \pm 1\rangle$ to $|1,2,0\rangle$ as highlighted in Fig. 4. Here, a definitive assignment of all transitions cannot be achieved. Nevertheless, one can plausibly assume that the strongest excitation channel $\mathrm{E}$ is related to the $|1,0,0\rangle$ state. The light red lines in Fig. 4 indicate excitation channels with a low transition probability based on selection rule considerations ${ }^{8}$ and calculations employed for CdS. ${ }^{16}$ However, a calculation of the oscillator strength of all these electronic as well as rotational-vibrational states is beyond the scope of the present work, as additionally, band-mixing effects would need to be considered in $\mathrm{GaN}$, as it was done for $\mathrm{CdS} .{ }^{16}$

\section{DISCUSSION}

As SIMS measurements show that our sample is characterized by oxygen and silicon concentrations below the SIMS detection limit, we relied on our PL and PLE analysis in order to infer their respective spectroscopic contribution. Our reasoning supporting the negligible contribution of oxygen in the GaN sample is threefold. First, the PL spectrum shown in Fig. 1 is dominated by the emission related to $\mathrm{Si}^{0} \mathrm{X}_{A}^{n=1}$, while only a very faint and spectrally undefined signal is observed for $\mathrm{O}^{0} \mathrm{X}_{A}^{n=1}$ about $900 \mu \mathrm{eV}$ lower in energy. ${ }^{8}$ As a side result, the TES spectra do not show any oxygenrelated spectral features as discussed in Sec. III A. In addition, the energy of the rotational-vibrational states of group I is typical for silicon with 1.18(1) $\mathrm{meV}$ as shown in Fig. 3 in agreement with the value of $1.2 \mathrm{meV}$ reported in the literature. ${ }^{8}$ As oxygen exhibits a larger $E_{l o c}$ value compared to silicon in $\mathrm{GaN}$, the corresponding energy of the group I rotational-vibrational states increases, ${ }^{13}$ leading to an $\sim 100 \mu \mathrm{eV}$ larger value as shown in PL spectra reported by Monemar et al. ${ }^{8}$

The PL (black) and PLE (red) spectra displayed in Fig. 3 show that the $\mathrm{Si}^{0} \mathrm{X}_{A}^{n=1}$ ground state transition at 3.47300(1) $\mathrm{eV}$ is accompanied by an additional spectral feature $\mathrm{S}$ that appears $430(10) \mu \mathrm{eV}$ lower in energy, while the electronic excitation channel A is situated 450(10) $\mu \mathrm{eV}$ higher in energy. In addition, the PLE spectrum at the energy position of the $\mathrm{S}$ line illustrated in Fig. 3 (purple) shows that the $\mathrm{Si}^{0} \mathrm{X}_{A}^{n=1}$ ground state and the spectral feature A form clear excitation channels, while contributions from rotational-vibrational excited states are absent.

If the $\mathrm{S}$ line were a bound exciton related to another impurity, then one would expect such rotational-vibrational excited states at an energy value close to $1.18(1) \mathrm{meV}$ in accordance with our observation for $\mathrm{Si}^{0} \mathrm{X}_{A}^{n=1}$ (see Fig. 3), because the energy of a rotational-vibrational excited state scales with the $E_{l o c}$ value of the particular bound exciton. ${ }^{13}$ For instance, even for the spectral feature A we can observe a rotational-vibrational state with an energy splitting of 1.16(1) $\mathrm{meV}$ in a corresponding PLE spectrum (not shown). Naturally, one could assume that every electronic excited state features its own rotational-vibrational state(s) similar to the electronic ground state transition. This observation along with our calculations supports that the feature A is indeed related to the energetically lowest lying electronic excited state of $\mathrm{Si}^{0} \mathrm{X}_{A}^{n=1}$ and not to another impurity. Hence, the PLE spectrum of the S line in Fig. 3 does not indicate an exciton transfer in between different impurity species. ${ }^{11}$ However, the origin of the $\mathrm{S}$ line and its relation to the $\mathrm{Si}^{0} \mathrm{X}_{A}^{n=1}$ ground state remains to be clarified. Generally, at least three possible origins of the S line come into mind as discussed in the following. The S line could either originate from an interaction with acoustic phonons, from a strain-induced splitting of the degenerate states of the $\mathrm{D}^{0} \mathrm{X}_{\mathrm{A}}$ center, or from an isotope effect in GaN.

\section{A. Isotope effect in GaN}

Two types of isotope effects can be distinguished for bound excitons leading to a shift of their emission. Either the isotopes of the dopant or the isotopes that constitute the host material can lead to ground state splittings of, e.g., a $\mathrm{D}^{0} \mathrm{X}_{\mathrm{A}}$ complex. Stable isotopes of the dopant silicon are $\mathrm{Si}^{A=28,29,30}$, with $\mathrm{Si}^{A=28}$ as the most common species on earth $(92.2 \%)$. However, $\mathrm{D}^{0} \mathrm{X}_{\mathrm{A}}$ centers with higher isotopic masses appear at higher energies in PL spectra, ${ }^{36}$ which nullifies their relevance for the interpretation of the $\mathrm{S}$ line. In addition, such isotopic effects are known to cause energy shifts far below the linewidths of our sample. ${ }^{36}$ In contrast, an isotope effect in the host material could indeed evoke energy shifts on an order of magnitude that could be resolved for our sample. ${ }^{37}$ First, an isotopic effect for nitrogen can be neglected as among the stable isotopes $\mathrm{N}^{A=14,15}$ the natural occurrence of $\mathrm{N}^{A=14}$ strongly dominates with $99.6 \%$. In contrast, two stable isotopes $\left(\mathrm{Ga}^{A=69,71}\right)$ exist for the cation lattice site of $\mathrm{GaN}$, exhibiting a natural occurrence of $60.1 \%$ and $39.9 \%$, respectively. However, similar to the first type of isotope effect, any heavier isotope introduces a $\mathrm{D}^{0} \mathrm{X}_{\mathrm{A}}$ shift to higher energies. Hence, even the second type of isotopic effect that directly affects the host material cannot explain the occurrence of the S line. Only the A line is situated in an energetic range that could be encompassed by an isotopic shift of a bound exciton as, e.g., reported by Zhang et al. for $\mathrm{Cd}^{A=110,112,114,116}$ in $\mathrm{CdS} .{ }^{37}$ However, the ratio for the natural occurrence of $\mathrm{Ga}^{A=69,71}(\sim 1.5: 1)$ contradicts the emission line intensity ratio we observe in Fig. 3 for $\mathrm{Si}^{0} \mathrm{X}_{A}^{n=1}$ and its companion A ( $\sim 3: 1)$. As a result, an isotope effect cannot straightforwardly explain the A or S lines in our spectra. To summarize, the A line is identified as an excited state of the $\mathrm{Si}^{0} \mathrm{X}_{A}^{n=1}$ ground state, while the nature of the S-line still remains to be identified.

\section{B. Strain-induced ground state splittings of a $D^{0} X_{A}$ complex}

At first glance, the splitting of the $\mathrm{X}_{\mathrm{A}}$ transition in $\mathrm{GaN}$ into an allowed $\Gamma_{5}$ orthoexciton and a forbidden $\Gamma_{6}$ paraexciton due to the $\Gamma_{9}$-symmetry of the topmost valence band appears as another possible explanation for the appearance of the $\mathrm{S}$ line. Paskov et al. ${ }^{38}$ have reported a $\Gamma_{5}-\Gamma_{6}$ splitting for the free A-exciton in $\mathrm{GaN}$ of 520(40) $\mu \mathrm{eV}$, which seems to compare well with the splitting of the S line [430(10) $\mu \mathrm{eV}]$ and its intensity ratio to the ground state. However, Thomas and Hopfield have pointed out that for wurtzite $\mathrm{CdS}^{15}$ such a $\Gamma_{5}-\Gamma_{6}$ splitting for a bound state can only appear for an ionized donor or acceptor bound exciton as their neutral 
counterparts always exhibit antiparallel spins for the two identical charges. Hence, no zero-field ground state splitting should occur for the $\mathrm{Si}^{0} \mathrm{X}_{A}^{n=1}$ complex. However, Reynolds et al. ${ }^{39}$ have observed a splitting of a $\mathrm{D}^{0} \mathrm{X}_{\mathrm{A}}$ complex in $\mathrm{ZnO}$ depending on which material ( $\mathrm{ZnS}$ or $\mathrm{ZnSe})$ they used as a starting material for the crystal growth. They concluded that using $\mathrm{ZnSe}$ evokes a particular strain distribution in the sample that affects the spin exchange coupling in the $\mathrm{D}^{0} \mathrm{X}_{\mathrm{A}}$ complex. Thus, under a magnetic field they could resolve a fourfold splitting of the $\mathrm{D}^{0} \mathrm{X}_{\mathrm{A}}$ transition, while under zero-field conditions only three components were observed-a situation very similar to our own observation, cf. Fig. 3 and Ref. 39. Indeed, the growth technique applied for our GaN substrate ${ }^{12}$ could explain such a strain induced $\mathrm{D}^{0} \mathrm{X}_{\mathrm{A}}$ splitting in our sample as the dislocation density varies over the surface area. In addition, one could expect that the underlying $\mathrm{Al}_{0.06} \mathrm{Ga}_{0.94} \mathrm{~N}$ layer affects the strain state of the analyzed $\mathrm{GaN}$ epilayer grown on top of it (see Sec. II). However, confocal micro Raman measurements performed with an excitation spot diameter of around $400 \mathrm{~nm}$ (not shown) reveal an $E_{2}^{\text {high }}$ mode position of $566.8(2) \mathrm{cm}^{-1}$ for our sample, which is very close to the relaxed value ${ }^{40}$ for bulk $\mathrm{GaN}$ of $567.0 \mathrm{~cm}^{-1}$. In addition, we find a full-width-at-half maximum for the $E_{2}^{\text {high }}$ mode of $2.8(3) \mathrm{cm}^{-1}$ at $300 \mathrm{~K}$, an almost lifetime-limited value for $\mathrm{GaN}^{41,42}$ pointing to minor strain fluctuations within the excitation spot diameter. Hence, strain fluctuations at the microand nano-scale appear to be small in our sample, making the observation of a strain-induced splitting of the $\mathrm{D}^{0} \mathrm{X}_{\mathrm{A}}$ unlikely, despite apparent similarities between the present $\mathrm{GaN}$ and $\mathrm{ZnO}^{39}$ PL spectra. The relevant PL signature of both materials shows a peak triplet under zero-field conditions that consists of the $\mathrm{S}$ line, the $\mathrm{Si}^{0} \mathrm{X}_{A}^{n=1}$ ground state, and the $\mathrm{A}$ line in our $\mathrm{GaN}$ sample. However, all strain-split components of such a $\mathrm{D}^{0} \mathrm{X}_{\mathrm{A}}$ center should feature rotational-vibrational excited states, which is the case for the $\mathrm{A}$ line and $\mathrm{Si}^{0} \mathrm{X}_{A}^{n=1}$ but not for the $\mathrm{S}$ line, cf. Fig. 3.

\section{Interaction of a $D^{0} X_{A}$ complex with acoustic phonons}

Figure 3 shows an additional peak triplet centered around $\mathrm{Si}^{0} \mathrm{X}_{B}^{n=1}$, which is very similar to our observation for $\mathrm{Si}^{0} \mathrm{X}_{A}^{n=1}$ along with the two PL and PLE lines named $\mathrm{S}$ and A. Accordingly, we will name the low- and high-energy parts of the peak triplet centered at $\mathrm{Si}^{0} \mathrm{X}_{B}^{n=1}$ the $\mathrm{S}^{\prime}$ and $\mathrm{A}^{\prime}$ lines, cf. Fig. 3. Interestingly, for $\mathrm{A}$ and $\mathrm{A}^{\prime}$ the splitting to their corresponding ground state is reduced from $450(10) \mu \mathrm{eV}$ to $360(30) \mu \mathrm{eV}$, while for $\mathrm{S}$ and $\mathrm{S}^{\prime}$ the splitting evolves from $430(10) \mu \mathrm{eV}$ to just $390(30) \mu \mathrm{eV}$. Regarding the $\mathrm{A}$ and $\mathrm{A}^{\prime}$ lines that each correspond to a $|0,1,0\rangle$ excited state, we believe that this subtle difference is caused by deviating averaged effective hole masses related to either the A- or B-valence band. Generally, the excitation channel linewidths of the peak triplet around $\mathrm{Si}^{0} \mathrm{X}_{B}^{n=1}$ are increased in comparison to $\mathrm{Si}^{0} \mathrm{X}_{A}^{n=1}$, which can tentatively be explained by a lifetime broadening due to hole conversion from the Bto the A-valence band under phonon emission. ${ }^{15}$ Shah et al. have observed a distinct spectral feature in the low energy flank of a neutral acceptor-bound exciton in $\mathrm{CdS}$ (the so-called $\mathrm{I}_{1}$ line) at low temperatures. ${ }^{43}$ Upon rising temperature, they observe a prominent, asymmetric broadening that they attribute to the transition from Stokes- to an antiStokes-process involving acoustic phonons. The S line in our spectra could also originate from such an interaction with acoustic phonons. However, the interaction of neutral donorbound excitons with acoustic phonons is much weaker compared to their acceptor counterparts. ${ }^{15}$ Hence, a similar temperature-dependent analysis under selective excitation in $\mathrm{GaN}$ proves challenging and must remain a task for future work. Generally, isoelectronic doping of our GaN sample with indium - a way to introduce disorder in the crystalintensifies the PL and PLE contributions of selected excited states of $\mathrm{Si}^{0} \mathrm{X}_{A}^{n=1}$, before a broadening sets in at higher doping concentrations at the onset of alloy formation $(\geq 0.01 \%$ indium content). Clearly, quadrupole transitions like $|0,1,0\rangle$ and $|0,1, \pm 1\rangle,{ }^{16}$ which match the excitation channels $\mathrm{A}$ and $\mathrm{C}$ in Fig. 4 intensify due to such an increase in disorder in the crystal. However, the $\mathrm{S}$ line also intensifies with rising indium content, which we tentatively attribute to disorderinduced phonon-confinement, boosting the exciton-phonon interaction as commonly observed for nanostructures. ${ }^{44-46}$

\section{SUMMARY}

In summary, we have presented a detailed analysis of the excited states related to the neutral silicon donor bound exciton $\left(\mathrm{Si}^{0} \mathrm{X}_{A}^{n=1}\right)$ in a high quality $\mathrm{GaN}$ epilayer. Based on PL and PLE results in conjunction with calculations, we could classify these excited states into rotational-vibrational and electronic states. For the latter class, we could not only identify excited states comprising a hole from the A-valence band, but also states with a hole from the B-valence band $\left(\mathrm{Si}^{0} \mathrm{X}_{B}^{n=1}\right)$ accompanied by their very own hierarchy of excited states. Our results show that great care must be taken when identifying bound excitons in $\mathrm{GaN}$ as even a single bound exciton is accompanied by a large set of excited states with prominent PL contributions depending on the particular excitation conditions. The key for the present identification of excitation channels in GaN was the low impurity concentration in our sample causing PL spectra to be only dominated by the $\mathrm{Si}^{0} \mathrm{X}_{A}^{n=1}$ emission, while PL contributions of the common oxygen donor were minor. In addition, we provided detailed insights into the rich structure of the fundamental absorption edge of GaN by not only identifying excited, $n=2$ states of the common exciton-polaritons $\left(\mathrm{X}_{A}^{n=2}, \mathrm{X}_{B}^{n=2}\right)$ and the related longitudinal-transverse split components, but also the corresponding Si-related bound state $\left(\mathrm{Si}^{0} \mathrm{X}_{A}^{n=2}\right)$. In conclusion, frequently reported linewidths of bound excitons in $\mathrm{GaN}$ are not necessarily characteristic for the particular sample as the wealth of excited states in $\mathrm{GaN}$ renders a meaningful linewidth analysis a challenging task that does not only require high-resolution spectroscopic equipment but also selective excitation. Hence, despite the nowadays widespread use of $\mathrm{GaN}$ in the industry, its careful spectroscopic analysis still remains challenging, as among other wurtzite materials like $\mathrm{CdS}$ and $\mathrm{ZnO}, \mathrm{GaN}$ represents the special case where small chemical shifts are paired with large, bound- 
excitonic linewidths and a rich optical signature related to exciton-polariton formation.

\section{ACKNOWLEDGMENTS}

Gordon Callsen acknowledges funding from the Marie Skłodowska-Curie action "PhotoHeatEffect" (Grant No. 749565) within the European Union's Horizon 2020 research and innovation program. In addition, we acknowledge support from the Deutsche Forschungsgemeinschaft (DFG, German Research Foundation) within the Collaborative Research Center "Semiconductor Nanophotonics" (CRC 787). The authors wish to thank Denis Martin for growing the analyzed GaN sample and Ian M. Rousseau for strong support with one of the experimental setups. Furthermore, we highly acknowledge the support of Axel Hoffmann, who has provided the experimental equipment for the highresolution PLE measurements and triggered fruitful discussions.

${ }^{1}$ B. K. Meyer, H. Alves, D. M. Hofmann, W. Kriegseis, D. Forster, F. Bertram, J. Christen, A. Hoffmann, M. Straßburg, M. Dworzak, U. Haboeck, and A. V. Rodina, Phys. Status Solidi 241, 231 (2004).

${ }^{2}$ D. C. Reynolds and T. C. Collins, Excitons, 1st ed. (Academic Press Inc., New York, 1981).

${ }^{3}$ G. Schramm, Phys. Status Solidi 120, K101 (1990).

${ }^{4}$ T. Agne, M. Dietrich, J. Hamann, S. Lany, H. Wolf, and T. Wichert, Appl. Phys. Lett. 82, 3448 (2003).

${ }^{5}$ J. L. Merz, H. Kukimoto, K. Nassau, and J. W. Shiever, Phys. Rev. B 6, 545 (1972).

${ }^{6}$ M. R. Wagner, J.-H. Schulze, R. Kirste, M. Cobet, A. Hoffmann, C. Rauch, A. V. Rodina, B. K. Meyer, U. Roeder, and K. Thonke, Phys. Rev. B 80, 205203 (2009).

${ }^{7}$ K. Kornitzer, T. Ebner, K. Thonke, R. Sauer, C. Kirchner, V. Schwegler, M. Kamp, M. Leszczynski, I. Grzegory, and S. Porowski, Phys. Rev. B 60, 1471 (1999).

${ }^{8}$ B. Monemar, P. P. Paskov, J. P. Bergman, A. A. Toropov, T. V. Shubina, T. Malinauskas, and A. Usui, Phys. Status Solidi B 245, 1723 (2008).

${ }^{9}$ G. Neu, M. Teisseire, P. Lemasson, H. Lahreche, N. Grandjean, F. Semond, B. Beaumont, I. Grzegory, S. Porowski, and R. Triboulet, Physica B 302-303, 39 (2001).

${ }^{10}$ J. A. Freitas, W. J. Moore, B. V. Shanabrook, G. C. B. Braga, S. K. Lee, S. S. Park, and J. Y. Han, Phys. Rev. B 66, 233311 (2002).

${ }^{11}$ P. J. Dean, D. C. Herbert, C. J. Werkhoven, B. J. Fitzpatrick, and R. N. Bhargava, Phys. Rev. B 23, 4888 (1981).

${ }^{12}$ K. M. Motoki, T. O. Kahisa, N. M. Atsumoto, M. M. Atsushima, and H. K. Imura, Jpn. J. Appl. Phys., Part 2 40, L140 (2001).

${ }^{13}$ B. K. Meyer, J. Sann, S. Eisermann, S. Lautenschlaeger, M. R. Wagner, M. Kaiser, G. Callsen, J. S. Reparaz, and A. Hoffmann, Phys. Rev. B 82, 115207 (2010)

${ }^{14}$ J. Gutowski, Phys. Rev. B 31, 3611 (1985)

${ }^{15}$ D. G. Thomas and J. J. Hopfield, Phys. Rev. 128, 2135 (1962).

${ }^{16}$ J. Puls, F. Henneberger, and J. Voigt, Phys. Status Solidi 119, 291 (1983).

${ }^{17}$ D. Labrie, T. Timusk, and M. L. W. Thewalt, Phys. Rev. Lett. 52, 81 (1984).
${ }^{18}$ M. L. W. Thewalt, D. Labrie, and T. Timusk, Solid State Commun. 53, 1049 (1985).

${ }^{19}$ A. Wysmolek, K. P. Korona, R. Stępniewski, J. M. Baranowski, J. Błoniarz, M. Potemski, R. L. Jones, D. C. Look, J. Kuhl, S. S. Park, and S. K. Lee, Phys. Rev. B 69, 157302 (2004).

${ }^{20}$ B. Šantic, C. Merz, U. Kaufmann, R. Niebuhr, H. Obloh, and K. Bachem, Appl. Phys. Lett. 71, 1837 (1997).

${ }^{21}$ B. Monemar, W. M. Chen, P. P. Paskov, T. Paskova, G. Pozina, and J. P. Bergman, Phys. Status Solidi B 228, 489 (2001).

${ }^{22}$ A. Wysmołek, M. Potemski, K. Pakuła, J. M. Baranowski, I. Grzegory, S. Porowski, G. Martinez, and P. Wyder, Phys. Rev. Lett. 91, 226404 (2003).

${ }^{23}$ S. Fischer, D. Volm, D. Kovalev, B. Averboukh, A. Graber, H. C. Alt, and B. K. Meyer, Mater. Sci. Eng. B 43, 192 (1997).

${ }^{24}$ A. V. Rodina, M. Dietrich, A. Göldner, L. Eckey, A. Hoffmann, A. Efros, M. Rosen, and B. Meyer, Phys. Rev. B 64, 115204 (2001).

${ }^{25}$ J. Sak, Phys. Rev. B 3, 3356 (1971)

${ }^{26}$ D. M. Larsen, Phys. Rev. B 9, 823 (1974).

${ }^{27}$ P. P. Paskov, T. Paskova, P. O. Holtz, and B. Monemar, Phys. Status Solidi A 201, 678 (2004).

${ }^{28}$ M. R. Wagner, G. Callsen, J. S. Reparaz, R. Kirste, A. Hoffmann, A. V. Rodina, A. Schleife, F. Bechstedt, and M. R. Phillips, Phys. Rev. B 88, 235210 (2013).

${ }^{29}$ K. R. Elliott, G. C. Osbourn, D. L. Smith, and T. C. McGill, Phys. Rev. B 17, 1808 (1978)

${ }^{30}$ C. Klingshirn, J. Guerts, A. Hoffmann, B. Meyer, and A. Waag, ZnO: From Fundamental Properties towards Novel Applications, 1st ed. (Springer, Berlin/Heidelberg, 2010).

${ }^{31}$ B. Gil, P. Bigenwald, M. Leroux, P. P. Paskov, and B. Monemar, Phys. Rev. B 75, 085204 (2007).

${ }^{32}$ W. Rühle and W. Klingenstein, Phys. Rev. B 18, 7011 (1978).

${ }^{33}$ G. Callsen, M. R. Wagner, T. Kure, J. S. Reparaz, M. Bügler, J. Brunnmeier, C. Nenstiel, A. Hoffmann, M. Hoffmann, J. Tweedie, Z. Bryan, S. Aygun, R. Kirste, R. Collazo, and Z. Sitar, Phys. Rev. B 86, 075207 (2012).

${ }^{34}$ S. Brochen, J. Brault, S. Chenot, A. Dussaigne, M. Leroux, and B. Damilano, Appl. Phys. Lett. 103, 032102 (2013).

${ }^{35}$ J. Gutowski, N. Presser, and I. Broser, Phys. Rev. B 38, 9746 (1988).

${ }^{36}$ M. Cardona and M. L. W. Thewalt, Rev. Mod. Phys. 77, 1173 (2005).

${ }^{37}$ J. M. Zhang, T. Ruf, R. Lauck, and M. Cardona, Phys. Rev. B 57, 9716 (1998).

${ }^{38}$ P. P. Paskov, T. Paskova, P. O. Holtz, and B. Monemar, Phys. Rev. B 64, 115201 (2001).

${ }^{39}$ D. C. Reynolds, D. C. Look, B. Jogai, and T. C. Collins, Phys. Rev. B 56, 13753 (1997).

${ }^{40}$ U. Haboeck, H. Siegle, A. Hoffmann, and C. Thomsen, Phys. Status Solidi C 0, 1710 (2003).

${ }^{41}$ C. Nenstiel, M. Bügler, G. Callsen, F. Nippert, T. Kure, S. Fritze, A. Dadgar, H. Witte, J. Bläsing, A. Krost, and A. Hoffmann, Phys. Status Solidi (RRL) 9, 716 (2015).

${ }^{42}$ T. Beechem and S. Graham, J. Appl. Phys. 103, 093507 (2008).

${ }^{43}$ J. Shah, R. F. Leheny, and W. F. Brinkman, Phys. Rev. B 10, 659 (1974).

${ }^{44}$ I. A. Ostapenko, G. Hönig, S. Rodt, A. Schliwa, A. Hoffmann, D. Bimberg, M. R. Dachner, M. Richter, A. Knorr, S. Kako, and Y. Arakawa, Phys. Rev. B 85, 081303(R) (2012).

${ }^{45}$ G. Callsen, G. M. O. Pahn, S. Kalinowski, C. Kindel, J. Settke, J. Brunnmeier, C. Nenstiel, T. Kure, F. Nippert, A. Schliwa, A. Hoffmann, T. Markurt, T. Schulz, M. Albrecht, S. Kako, M. Arita, and Y. Arakawa, Phys. Rev. B 92, 235439 (2015).

${ }^{46}$ F. Rol, S. Founta, H. Mariette, B. Daudin, L. S. Dang, J. Bleuse, D. Peyrade, J. M. Gérard, and B. Gayral, Phys. Rev. B 75, 125306 (2007). 